\title{
The importance of Noether's theorem for the way we think today
}

\author{
\&C. Quigg 吕7-12-2021 ه http://www.primapagina.sif.it/article/1424
}

In the summer of 1918, German mathematician Emmy Noether published the two-part theorem that now bears her name, establishing profound connections between symmetries and conservation laws and between symmetries and interactions. These insights exert a pervasive influence in physics; they give meaning to conservation laws that elevates them beyond useful empirical rules, and they underlie all our theories of the fundamental interactions. In Noether's later career, her papers, lectures, and personal interactions with students and colleagues drove the development of abstract algebra, establishing her in the pantheon of twentieth-century mathematicians.

A recent CERN Colloquium traced her path from her birthplace in Erlangen through Göttingen to a brief but happy exile at Bryn Mawr College in Pennsylvania, illustrating the importance of "Noether's Theorem" for the way we think today. An accompanying essay provides an entrée into the literature about Noether's life and work.

Emmy Noether's great innovation was to explore what happens when the equations that govern physical processes remain unchanged in form when transformed by symmetry operations. For example, the notion that Nature's laws do not privilege a particular time or place leads to the conclusion that the total energy and momentum are fixed quantities. These famous conservation laws are thus not merely convenient empirical rules; they arise from symmetries of the laws of motion under translations-shifts-in time or space. Applied to these situations, Noether's mathematical formulation demystified rules of physics that had grown up over many years.

What if the symmetry is not a simple shift, but a transformation different in degree at different points in space and time? If we impose this requirement on a theory without interactions, it forces us to modify the equations of physics to include interactions of a very particular character. Hermann Weyl gave a specific example, deriving electromagnetism by imposing a symmetry on the quantum-mechanical wave function. The full power of the theorem only became evident to physicists in 1954 when Chen Ning Yang and Robert Mills based a theory of nuclear forces on the isospin symmetry that relates proton and neutron. Their attempt did not describe Nature, but the principle that symmetries dictate interactions would later give rise to the electroweak theory and to quantum chromodynamics, the theory of interactions among quarks and gluons. General relativity similarly follows from a symmetry principle.

Emmy Noether's story is one of obstacles to the higher education of women in early twentieth-century Germany, the exclusion of women from academic posts until the nineteen-twenties, and the Nazi purge of those deemed "undesirable" in 1933. It is also a tale of her talent, persistence, and generous spirit. Mathematicians revere Noether as the Mother of Modern Algebra. Physicists are indebted to her for the theorem that invites us to see symmetries, conservation laws, and interactions-Nature-in a unifying light.

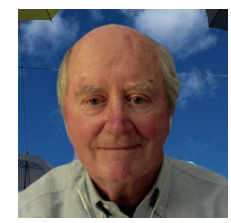

Chris Quigg - Distinguished Scientist Emeritus at the Fermi National Accelerator

Laboratory, his research ranges from heavy quarks through cosmic neutrinos. His work on electroweak symmetry breaking and supercollider physics, which was recognized by the 2011 J.J. Sakurai Prize of the American Physical Society for outstanding achievement in particle theory, charted the course for exploration at Fermilab's Tevatron and CERN's Large Hadron Collider. 